\title{
Characteristics of intraretinal deposits in acute central serous chorioretinopathy
}

This article was published in the following Dove Press journal:

Clinical Ophthalmology

2 April 2014

Number of times this article has been viewed

\section{Andrea M Plateroti \\ Matthew T Witmer \\ Szilárd Kiss \\ Donald J D'Amico}

Department of Ophthalmology, Weill Cornell Medical College, New York, NY, USA
Correspondence: Matthew T Witmer

Weill Cornell Medical College,

Department of Ophthalmology,

I 305 York Avenue - I I th floor.

New York, NY 1002I, USA

$\mathrm{Tel}+\mathrm{I} 6469622020$

Fax + I 6469620602

Email maw2052@med.cornell.edu
Purpose: To describe the temporal and spatial characteristics of intraretinal deposits in patients with acute central serous chorioretinopathy (CSC) using spectral domain optical coherence tomography (OCT).

Materials and methods: We retrospectively reviewed the medical records of all patients that presented with acute CSC to Weill Cornell Medical College from January 2012 to May 2013. Acute CSC was defined as a diagnosis of CSC within 4 months of the onset of symptoms. Only one eye per patient was included in the study. Each patient was imaged with spectral domain OCT at the initial office visit. The decision to reimage these patients was made by the treating physician.

Results: A total of 25 patients ( 25 eyes; 17 men and eight nonpregnant women) were included in this review. Seven of 25 patients $(28 \%)$ demonstrated intraretinal deposits within the outer plexiform layer during the initial OCT, with deposits appearing as early as the same day as the onset of symptoms. A total of 25 of 25 patients (100\%) demonstrated intraretinal deposits in the outer nuclear layer upon initial (76\%) or follow-up OCT, as early as 2 days after the onset of symptoms. A total of 24 of 25 patients (96\%) demonstrated deposits in the external limiting membrane upon a follow-up OCT, as early as 7 days from symptoms appearing. A total of 24 of 25 patients $(96 \%)$ developed intraretinal deposits in the inner segment/outer segment layer upon follow-up OCT, appearing as early as 14 days after symptom onset. At the time of resolution of subretinal fluid, 20 of 25 patients $(80 \%)$ demonstrated intraretinal deposits.

Conclusion: Intraretinal deposits are present in the outer retinal layers in patients with acute $\mathrm{CSC}$, with the deposits appearing progressively deeper within the retina as the condition evolves. Upon resolution of subretinal fluid, the deposits slowly resolve.

Keywords: acute central serous chorioretinopathy, intraretinal deposits, spectral domain OCT

\section{Introduction}

Central serous chorioretinopathy (CSC) is a disorder characterized by serous retinal detachment and/or retinal pigmented epithelial (RPE) detachments associated with a thickened choroid. These changes are most often confined to the macula and associated with leakage of fluid through the RPE into the subretinal space. ${ }^{1} \mathrm{CSC}$ is a common clinical diagnosis, especially in young to middle-aged adults, ${ }^{2}$ and has been associated with the use of several medications (chronic corticosteroid therapy) and several systemic conditions (hypertensive diseases). Patients usually present with blurred vision, metamorphopsia, micropsia ${ }^{3}$ or a relative central scotoma. ${ }^{4}$

Optical coherence tomography (OCT) has the ability to evaluate intraretinal changes and abnormalities, ${ }^{1,5}$ with a resolution of several microns. OCT imaging 
can noninvasively identify the presence and extent of subretinal fluid (SRF) and pigmented epithelium detachments, and it is also useful for assessing the resolution of SRF and morphological changes during normal disease progression. With the new generation of spectral domain OCT (SD-OCT), topographic changes in CSC can be visualized with two- and three-dimensional reconstructions; it also offers the ability to accurately evaluate volumetric measurements. ${ }^{6}$

Many authors have reported the presence of amorphous material in patients with CSC, which is usually located anterior to the RPE. ${ }^{7-11}$ These deposits, however, are also located within the retina during the course of this condition. The aim of this study was to describe the location and duration of these intraretinal deposits in patients with acute CSC using SD-OCT and the correlation of these deposits with the trend of the disease.

\section{Materials and methods}

We retrospectively reviewed the medical records of 25 patients ( 25 eyes) (17 men and 8 nonpregnant women) who presented to Weill Cornell Medical College from January 2012 to May 2013 and were diagnosed with acute CSC. Acute CSC was defined as the presence of a serous detachment of the macular neurosensory retina upon OCT, focal leakage of fluorescein on fluorescein angiography and the onset of such symptoms as metamorphopsia (94\%), decreased visual acuity (VA) (90\%), decreased color vision (43\%), and micropsia (37\%) within the previous 4 months.
Eyes with other macular abnormalities were excluded. Measurements of the best-corrected visual acuity (BCVA), dilated fundus examination, and SD-OCT with a Heidelberg Spectralis $^{\circledR}$ (Heidelberg Engineering, Heidelberg, Germany) were performed at every visit. Fluorescein angiography was performed at the baseline visit. The treating physician determined the follow-up schedule of all patients.

The OCT scans of each subject were evaluated at the initial visit and at each follow-up visit for the presence and characteristics of intraretinal deposits.

\section{Results}

The mean age of men included in the study was 41 (range 26-69) years, and their BCVA was 0.65, with a standard deviation (SD) 0.33 . The mean age of women included in the study was 54 (range 40-72) years, and their mean BCVA was 0.73 (SD 0.17). Seven of 25 patients (28\%) presented with intraretinal deposits within the outer plexiform layer on their OCT during their initial visit. These deposits appeared as early as the same day as the onset of symptoms (average duration of symptoms was 4 days, SD 2.83, range 1-7). Nineteen of $25(76 \%)$ patients demonstrated intraretinal deposits in the outer nuclear layer (ONL) upon their initial OCT. These deposits appeared as early as 2 days after the onset of symptoms (average duration of symptoms was 37.8 days, SD 34.97, range 2-120). At some point during follow-up, 100\% of patients demonstrated intraretinal deposits on the OCT in the ONL. A total of 24 of 25 patients $(96 \%)$ demonstrated deposits in the external limiting membrane at some point

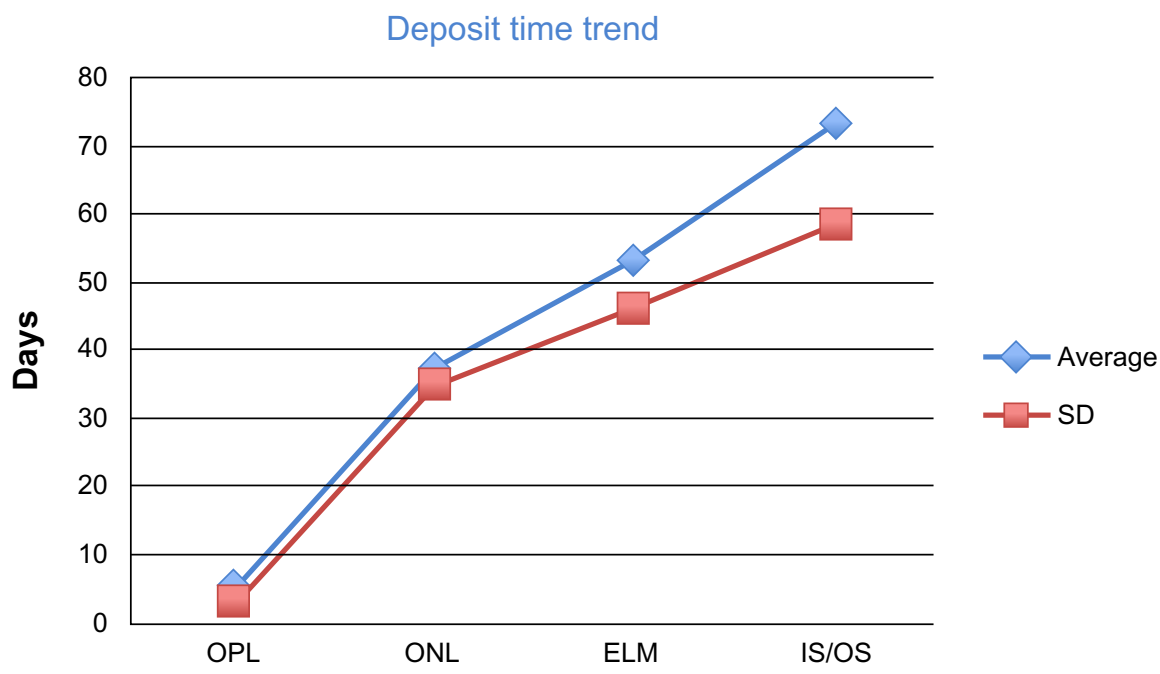

Figure I Number of days plotted against the four main involved layers. The number of days is represented on the vertical axis; the horizontal axis shows the four layers. The square dots in the chart indicate the average (blue dots) and the standard deviation (red dots) of days since the onset of symptoms in which deposits showed up in one of the involved layers. The figure can help better understanding of the time progression of deposit through the layers.

Abbreviations: OPL, outer plexiform layer; ONL, outer nuclear layer; ELM, external limiting membrane; IS/OS, inner segment/outer segment; SD, standard deviation. 
Table I Progression of intraretinal deposits in acute central serous chorioretinopathy patients

\begin{tabular}{lllll}
\hline Patient & \multicolumn{4}{l}{ Layer of intraretinal deposits } \\
\cline { 2 - 5 } & OPL & ONL & ELM & IS/OS \\
\hline 1 & 2 days & 2 days & 109 days & 109 days \\
2 & & 47 days & 47 days & 70 days \\
3 & 7 days & 7 days & 118 days & 118 days \\
4 & 1 day & 5 days & 28 days & 28 days \\
5 & & 48 days & 48 days & 115 days \\
6 & & 30 days & 30 days & 105 days \\
7 & & 7 days & 7 days & 97 days \\
8 & 2 days & 64 days & 64 days & 64 days \\
9 & 7 days & 7 days & 14 days & 76 days \\
10 & 2 days & 7 days & & \\
11 & 7 days & 7 days & 7 days & 55 days \\
12 & & 15 days & 15 days & 70 days \\
13 & & 14 days & 14 days & 110 days \\
\hline
\end{tabular}

Abbreviations: OPL, outer plexiform layer; ONL, outer nuclear layer; ELM, external limiting membrane; IS/OS, inner-segment/outer-segment junction.

during their follow-up OCT. These deposits appeared as early as 7 days from the onset of symptoms (average duration of symptoms was 53.17 days, SD 46.18, range 7-120). A total of 24 of 25 patients ( $96 \%$ ) developed intraretinal deposits in the inner-segment/outer-segment layers at some point during their follow-up. These deposits appeared as early as 14 days after the onset of symptoms (average duration of symptoms was 73.46 days, SD 58.98, range 14-120). At the time of resolution of SRF, 20 of 25 patients $(80 \%)$ demonstrated intraretinal deposits.

Thanks to multiple OCT scans, the location of the intraretinal deposits followed a progression to deeper layers of the retina during follow-up. Table 1 demonstrates 13 patients where it was possible to observe the evolution of the location
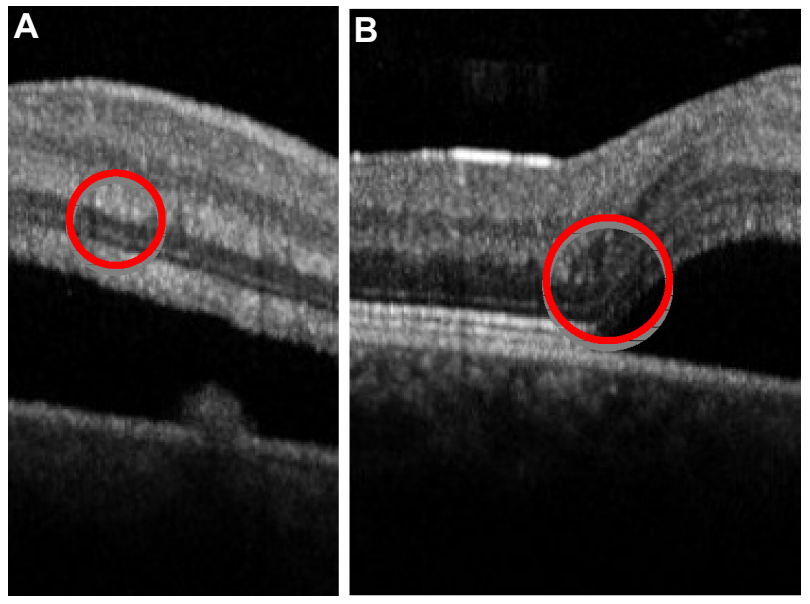

Figure 2 (A and B) Optical coherence tomography of a patient (patient I) with acute central serous chorioretinopathy, taken 2 days after the onset of symptoms. The red circles indicate intraretinal deposits (small white dots) inside the outer plexiform layer $(\mathbf{A})$ and the outer nuclear layer $(\mathbf{B})$. of intraretinal deposits over time. The characteristics of intraretinal deposits are better clarified in Figure 1, which demonstrates how they followed a temporal and spatial trend towards their appearance in deeper layers of the retina as the disease progressed.

The OCT images of Figures 2 and 3, taken from two different patients, demonstrate how these deposits appear in the deeper retinal layers as the duration of the disease increases. Figure 2A shows the OCT of a patient 2 days after the onset of symptoms. There are traces of intraretinal deposits in the outer plexiform layer. In the same OCT, deposits were also found in the ONL (Figure 2B). Figure 3A represents an OCT taken 10 days after symptom onset of a different patient, and shows intraretinal deposits in the external limiting membrane. Figure $3 \mathrm{~B}$ was taken on the same patient as Figure 2A, 15 days after the beginning of symptoms. SRF was completely resolved, but the red arrows indicate how deposits were still present in the inner-segment/outer-segment layer.

\section{Discussion}

The role of OCT as a noninvasive way of imaging the retina is well known. ${ }^{12-17}$ Several papers have described OCT findings in eyes with CSC and have documented the presence of subretinal or supra-RPE deposits, but very few reports discuss intraretinal deposits. ${ }^{1,11,18,19}$ This study demonstrates the evolution of intraretinal deposits as they appear more deeply within the outer retina as the disease progresses.

The intraretinal deposition described in this study appears to be a primary manifestation of CSC, but it differs in character and location from that seen in choroidal neovascularization (CNV). In fact, in acute stages of CNV, the two most common materials deposited under the retina
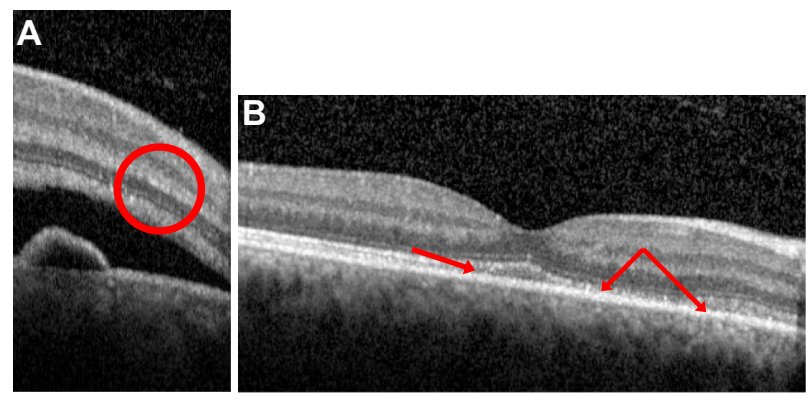

Figure 3 Optical coherence tomography of a patient (patient 12) with acute central serous chorioretinopathy, taken 10 (A) and 15 days (B) after the onset of symptoms. The red circle and red arrows indicate intraretinal deposits (small white dots), respectively, inside the external limiting membrane $(\mathbf{A})$ and the innersegment/outer-segment junction (B). (B) The subretinal fluid is completely resolved, but some deposits are still present even after this resolution. 
are blood and lipid. Blood is most commonly seen near the area of $\mathrm{CNV}$, while lipid is more common at the rim of the detachment at the outer-segment layer. ${ }^{8}$ The distribution of material in this study appears to be limited to the area of current or prior serous detachment.

The composition of the intraretinal deposits is not well established. Histopathological studies ${ }^{20,21}$ have shown the presence of fibrin inside the deposits and of a high protein content within the SRF. The material does not appear to be lipid; the patients in this and previous studies ${ }^{8}$ did not appear to have systemic inflammatory disease or evidence of ocular inflammation.

The pathogenesis of CSC has been hypothesized to be the dysfunction of the RPE cells, perhaps in the ion-pumping mechanism. ${ }^{22,23}$ Similarly, we hypothesize that the intraretinal deposits may accumulate secondary to the inability of the RPE cells to pump the highly proteinaceous SRF from the intraretinal and subretinal spaces.

In conclusion, we have shown a chronological trend to the appearance of intraretinal deposits in acute CSC that is dependent on the duration and onset of symptoms. The understanding of this process may be of clinical value in allowing physicians to better understand the staging of CSC and helping them in terms of diagnosing, monitoring, and deciding when to treat patients with this condition.

\section{Disclosure}

The authors report no conflicts of interest in this work.

\section{References}

1. Nicholson B, Noble J, Forooghian F, Meyerle C. Central serous chorioretinopathy: update on pathophysiology and treatment. Surv Ophthalmol. 2013;58(2):103-126.

2. Nair U, Ganekal S, Soman M, Nair K. Correlation of spectral domain optical coherence tomography findings in acute central serous chorioretinopathy with visual acuity. Clin Ophthalmol. 2012;6: 1949-1954.

3. Wang M, Munch IC, Hasler PW, Prünte C, Larsen M. Central serous chorioretinopathy. Acta Ophthalmol. 2008;86(2):126-145.

4. Spaide RF, Campeas L, Haas A, et al. Central serous chorioretinopathy in younger and older adults. Ophthalmology. 1996;103(12):2070-2079.

5. Iida T, Hagimura N, Sato T, Kishi S. Evaluation of central serous chorioretinopathy with optical coherence tomography. Am J Ophthalmol. 2000;129(1):16-20.

Clinical Ophthalmology

\section{Publish your work in this journal}

Clinical Ophthalmology is an international, peer-reviewed journal covering all subspecialties within ophthalmology. Key topics include: Optometry; Visual science; Pharmacology and drug therapy in eye diseases; Basic Sciences; Primary and Secondary eye care; Patient Safety and Quality of Care Improvements. This journal is indexed on Submit your manuscript here: http://www.dovepress.com/clinical-ophthalmology-journal
6. Stock G, Ahlers C, Sayegh R, et al. [Three-dimensional imaging in central serous chorioretinopathy]. Ophthalmologe. 2008;105(12): 1127-1134. German.

7. Hirami Y, Tsujikawa A, Sasahara M, et al. Alterations of retinal pigment epithelium in central serous chorioretinopathy. Clin Experiment Ophthalmol. 2007;35(3):225-230.

8. Ie D, Yannuzzi LA, Spaide RF, Rabb MF, Blair NP, Daily MJ. Subretinal exudative deposits in central serous chorioretinopathy. Br J Ophthalmol. 1993;77(6):349-353.

9. Kon Y, Iida T, Maruko I, Saito M. The optical coherence tomographyophthalmoscope for examination of central serous chorioretinopathy with precipitates. Retina. 2008;28(6):864-869.

10. Ojima Y, Hangai M, Sasahara M, et al. Three-dimensional imaging of the foveal photoreceptor layer in central serous chorioretinopathy using high-speed optical coherence tomography. Ophthalmology. 2007;114(12):2197-2207.

11. Wang M, Sander B, la Cour M, Larsen M. Clinical characteristics of subretinal deposits in central serous chorioretinopathy. Acta Ophthalmol Scand. 2005;83(6):691-696.

12. Drexler W, Sattmann H, Hermann B, et al. Enhanced visualization of macular pathology with the use of ultrahigh-resolution optical coherence tomography. Arch Ophthalmol. 2003;121:695-706.

13. Goebel W, Kretzchmar-Gross T. Retinal thickness in diabetic retinopathy: a study using optical coherence tomography (OCT). Retina. 2002;22;759-767.

14. He SZ, Wang W, Li XL, Tang R. [Optical coherence tomography features of central exudative chorioretinopathy]. Zhonghua Yan Ke Za Zhi. 2003;39:669-672. Chinese.

15. Hee MR, Puliafito CA, Duker JS, et al. Topography of diabetic macular edema with optical coherence tomography. Ophthalmology. 1998;105:360-370.

16. Iida T, Yannuzzi LA, Spaide RF, Borodoker N, Carvalho CA, Negrao S. Cystoid macular degeneration in chronic central serous chorioretinopathy. Retina. 2003;23(1):1-7.

17. Kamppeter B, Jonas JB. Central serous chorioretinopathy imaged by optical coherence tomography. Arch Ophthalmol. 2003;121(5):742-743.

18. Kim HC, Cho WB, Chung H. Morphologic changes in acute central serous chorioretinopathy using spectral domain optical coherence tomography. Korean J Ophthalmol. 2012;26(5):347-354.

19. Song IS, Shin YU, Lee BR. Time-periodic characteristics in the morphology of idiopathic central serous chorioretinopathy evaluated by volume scan using spectral-domain optical coherence tomography. Am J Ophthalmol. 2012;154(2):366-375.

20. De Venecia G. Fluorescein angiographic smoke stack. Case presentation at Verhoeff Society Meeting; Washington; April 24-25, 1982.

21. Ikui H. [Histological examination of central serous retinopathy]. Nihon Ganka Kiyo. 1969;20(11):1035-1041. Japanese.

22. Negi A, Marmor M. Experimental serous retinal detachment and focal pigment epithelial damage. Arch Ophthalmol. 1984;102(3):445-449.

23. Spitznas M. Pathogenesis of central serous retinopathy: a new working hypothesis. Graefes Arch Clin Exp Ophthalmol. 1986; 224(4):321-324.
PubMed Central and CAS, and is the official journal of The Society of Clinical Ophthalmology (SCO). The manuscript management system is completely online and includes a very quick and fair peer-review system, which is all easy to use. Visit http://www.dovepress.com/ testimonials.php to read real quotes from published authors. 